\title{
Nontarget objects can influence perceptual processes during object recognition
}

\author{
MARK E. AuCKLAND \\ University of Southampton, Southampton, England \\ KYLE R. CAVE \\ University of Massachusetts, Amherst, Massachusetts \\ AND \\ Nick DONNELLY \\ University of Southampton, Southampton, England
}

\begin{abstract}
Previous experiments have shown that objects are recognized more readily in a semantically consistent visual context. However, the benefit from context could be explained by response bias, and may not reflect the influence of context on the perceptual processes of recognition. We conducted a six-alternative forced-choice experiment to measure semantic and perceptual errors. A target object appeared briefly, surrounded by four context objects. The target was more accurately identified when the context consisted of objects semantically related to the target. The large number of semantic errors, which increased when the context presentation preceded the target, showed that response bias did account for a proportion of the context effect. Nevertheless, significant facilitation was still present after a bias correction. Recognition of an object can be affected by context not only when it is embedded in a coherent naturalistic scene, but also when it is simply near other related objects. Materials associated with this article may be accessed at www.psychonomic.org/archive.
\end{abstract}

Palmer (1975), Biederman (1981), and Davenport and Potter (2004) have shown that objects are recognized more readily when they appear within semantically consistent surroundings. Hollingworth and Henderson (1999) identified three possible explanations for this facilitation. In description enhancement, context enriches perceptual encoding, either by enhancing perceptual processing directly, or by identifying emergent features formed by combinations of objects (Biederman, 1981). Criterion modulation affects the matching of perceptual data against stored memory representations. Recognition thresholds are lowered (or activation levels raised) for memory representations that are consistent with specific contexts (see, e.g., Friedman, 1979; Kosslyn, 1994; Palmer, 1975). Description enhancement and criterion modulation both propose that context information is used prior to the completion of perceptual processing. The final explanation offered by Hollingworth and Henderson (1998), functional isolation, suggests that context information is used to make an informed guess after the perceptual processing. This "postperceptual" guess, based on context and not on perceptual information about the object itself, is defined here as response bias.

Hollingworth and Henderson (1998) provided evidence supporting the functional isolation hypothesis. They showed that Biederman's (1981) finding was contingent on computing false alarm rates across catch trials on semantically consistent and inconsistent trials. When false alarm rates were computed separately for target-present and catch trials (Experiments 2-4), the context effect found in this paradigm was revealed as an effect of response bias and not perceptual sensitivity. In addition, when a twoalternative forced-choice (2AFC) discrimination task was used that did not allow for effects of response bias, no effect of semantic consistency was found (Experiment 4). In summary, Hollingworth and Henderson's (1998) findings challenge the empirical basis of scene context effects and question the evidence used to support description enhancement and criterion modulation accounts.

However, if there was facilitation from criterion modulation, it may not have been detected in some of Hollingworth and Henderson's $(1998,1999)$ experiments. Criterion modulation utilizes both perceptual and contextual information, being most effective when a target that is consistent with the current context must be distinguished from a perceptually similar alternative object that is not. In the 2AFC tasks used by Hollingworth and Henderson $(1998,1999)$, both the target and the incorrect choice were members of the same contextual group and were influenced by similar contextual factors. Having two choices

K. R. Cave, kcave@psych.umass.edu 
from the same contextual group eliminates any advantage from guessing based solely on context, but it can also prevent the detection of the effects of criterion modulation, because the advantage from context favors the incorrect choice as well as the target. The effects of criterion modulation are more likely to be measurable if the choices include a target that benefits from context and an incorrect choice that does not, because under these circumstances, context can exert a stronger effect on the responses.

In some of Hollingworth and Henderson's $(1998,1999)$ experiments, rather than choosing one of two objects, participants saw a single object name and decided whether that object was present. These experiments would also be unlikely to detect criterion modulation if the objects whose names were used to allow comparisons of performance across items inside and outside the contextual group were not physically/perceptually similar to the target.

Even if contextual facilitation could have been measured in Hollingworth and Henderson's $(1998,1999)$ studies, the effects might have been obscured in some of those experiments, in which the target and distractor names were presented after the scene disappeared. Davenport and Potter (2004) noted that in the context-inconsistent trials, participants could deduce the target before the name was shown because it would have been the "odd object out" (e.g., a food mixer in a farmyard). Attention might then have been preferentially allocated to this odd one out after it was detected. Consequently, the postcue procedure may have inflated performance in inconsistent relative to consistent conditions, providing inconsistent-context targets with an asymmetrical advantage. This would account for the better detection of targets in semantically inconsistent conditions in the postview condition of Hollingworth and Henderson's (1998) Experiment 3 (and the nonsignificant effect in Experiment 4).

In summary, Hollingworth and Henderson's (1998, 1999) findings are consistent with the functional isolation hypothesis of contextual effects in object recognition. However, because of the two issues described above, we cannot discount with confidence the criterion modulation hypothesis.

The experiment reported here tested whether response bias alone could explain scene context effects generated by nontarget objects. The opportunities for context effects in visual object recognition are very rich in comparison with the word superiority studies that employed a $2 \mathrm{AFC}$ paradigm. To produce a fuller picture of the nature of the context effects, the present experiment included a 6AFC paradigm to examine both perceptual and semantic errors and control response bias. Targets were presented at fixation to remove effects of spatial uncertainty and of guessing which target would be cued. The functional isolation hypothesis predicts that the number of perceptual and semantic errors made when identifying an object within a consistent context will not be significantly different (after correction) from those made when identifying an object within an inconsistent context. However, if a context effect is found in this recognition task after correcting for response bias, this would support criterion modulation.
The stimuli in this experiment were arrays of color photos, with the target object located in the center of the screen and surrounded by four context objects. Previous work has suggested that contextual stimuli can be maintained in the iconic memory (Auckland, 2005), so contexts were presented simultaneously with, or before, targets $(0 \mathrm{msec}$, $-52 \mathrm{msec}$, or $-104 \mathrm{msec}$ ) to examine whether additional processing of nontargets increased the context effect. The search task used by Hollingworth and Henderson (1998, 1999) made it likely that nontargets received visual attention. To replicate this attentional allocation without the complications of search, we used an exogenous cue to ensure that participants directed attention to the nontargets as well as to the known location of the target. This cue consisted of a small circle that replaced the fixation cross, and then expanded at a steady rate from fixation, drawing attention outward. Because there was no fixation point other than the expanding circle, attention was allocated reflexively to the circle (Jonides, 1981; Müller \& Rabbitt, 1989; Turatto et al., 2000) and spread across the entire display area.

\section{METHOD}

\section{Participants}

Sixty undergraduates participated in one 30 -min session for course credits or for $£ 3.00$. All of the participants reported having normal or corrected-to-normal visual acuity and English as a first language. None had previously seen the pictures.

\section{Apparatus and Stimuli}

A Macintosh Power PC G4 400-MHz computer with a 19-in. ProNitron monitor (13-msec screen refresh) controlled stimulus presentation and response acquisition. The participants were seated $60 \mathrm{~cm}$ from the screen in a dimly lit room. Each object stimulus was a digital color photograph, of which the majority could be fitted within a circle of 3-cm radius (visual angle $2.68^{\circ}$ ), displayed on a white background. There were a total of 170 stimuli, organized into 34 context groups of 5 semantically related objects each (see www.psychonomic.org/ archive), with each group paired with another. Context-consistent trials drew targets and nontargets from the same context group, and contextinconsistent trials drew nontargets from the alternative context group within the pair. In a survey of 10 participants, all of the objects were rated highly for familiarity, and the context groups were rated highly semantically consistent. Targets were always placed at the center of the screen, and the nontargets were placed surrounding the target, one in each quadrant, within a $10.82-\mathrm{cm}$ radius (visual angle $10.26^{\circ}$ ) from the center. There was no stimulus overlap (see Figure 1).
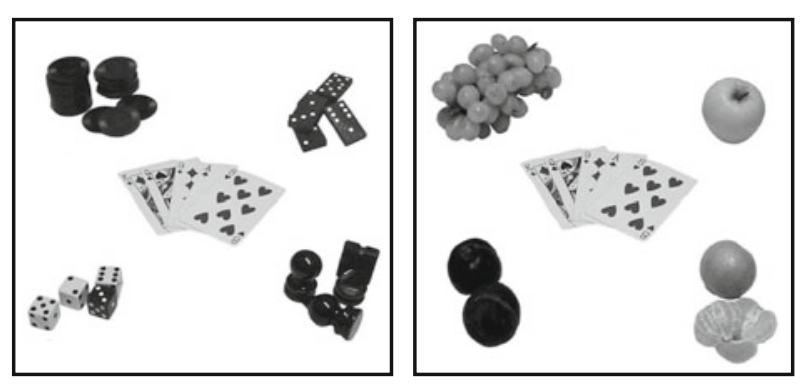

Figure 1. A target (playing cards) among context-consistent distractors (left) and among context-inconsistent distractors (right). 


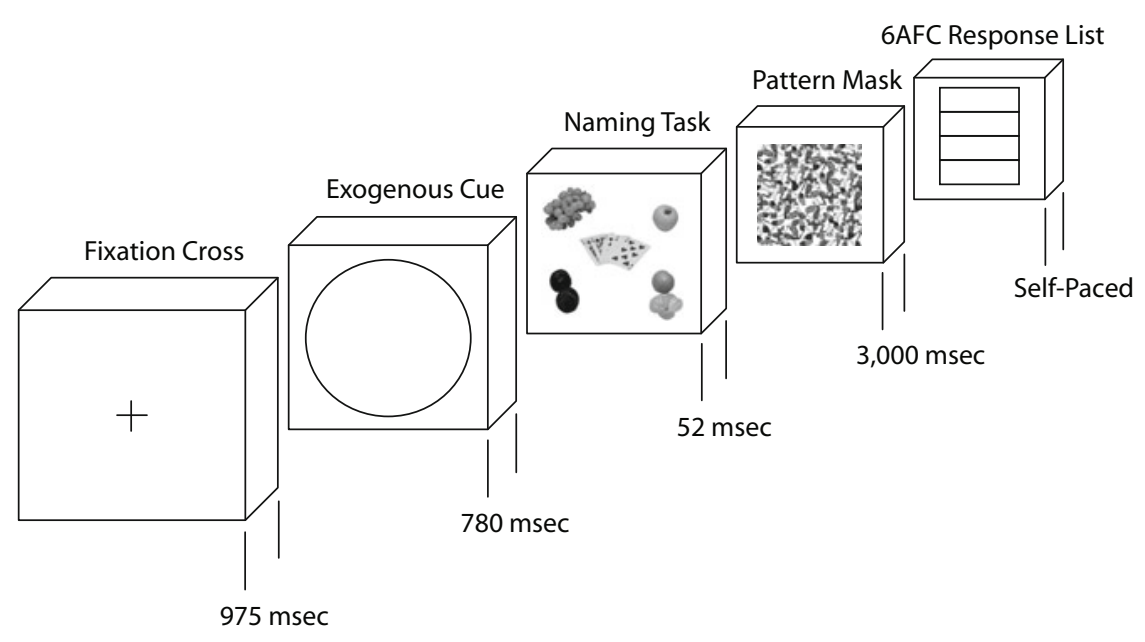

Figure 2. The progress of a single simultaneous target/context display trial.

\section{Procedure}

Figure 2 shows the sequence of events in a trial. All of the trials began with a fixation cross at the center of the screen for $975 \mathrm{msec}$. After removal of the cross, the outline of a circle expanded outward from the center, at a steady rate, until it reached a radius of visual angle $10.26^{\circ}$ after $780 \mathrm{msec}$. In simultaneous trials (stimulus onset asynchrony $[\mathrm{SOA}]=0 \mathrm{msec}$ ), the offset of the expanding cue was immediately followed by the onset of a target object and context together (see Figure 1), which was displayed for $52 \mathrm{msec}$. On trials in which the context stimulus appeared prior to the target ( $\mathrm{SOA}=$ $-52 \mathrm{msec}$ or $-104 \mathrm{msec}$ ), the nontarget objects appeared alone on the offset of the circle cue for $52 \mathrm{msec}$. These nontargets were arranged as in Figure 1, but without the target object at the center. In the $-52-\mathrm{msec}$ display condition, the offset of the context stimulus was immediately followed by the onset of the target object stimulus. In the -104-msec display condition, the offset of the context stimulus was followed by a blank screen display of $52 \mathrm{msec}$ before the display of the target. In both of these conditions, a target appeared at the center of the screen for $52 \mathrm{msec}$. In every condition, the target offset was followed by the onset of a multicolored mask that covered the entire display area for $1,000 \mathrm{msec}$.

At the offset of the visual mask, the 6AFC response display, consisting of a list of 6 object names, appeared. Three of the choices in the list were $(a)$ the target, $(b)$ an object perceptually similar to the target (but not semantically related), and (c) an object semantically related to the context (but not perceptually similar to the target).

With these choices, perceptual errors and semantic errors can be distinguished and used as a basis for detecting and correcting for response bias. However, a $3 \mathrm{AFC}$ response allows participants to deduce the context type of the previously viewed object array and modify their response strategy accordingly. For example, the target would be semantically related to the semantic error choice on a context-consistent trial but not on a context-inconsistent trial. Consequently, participants could deduce that a trial was context consistent if 2 of the choices were semantically related, and could limit their options to these 2 choices. To address this problem, we therefore provided 3 additional choices. These choices were $(d)$ an object physically similar to choice $c,(e)$ an object with no relationship to any previous choice (for context-consistent trials), or semantically related to choice $b$ (for context-inconsistent trials), and $(f)$ an object physically similar to choice $e$.

These additional options ensured that there were always three physically similar choice pairs, and one semantically related choice pair in every trial, regardless of context type. The six choices were listed vertically, and their order was randomized. The participants chose one using a mouse, with auditory feedback after incorrect responses.
Despite these controls, there was still an opportunity for guessing: Participants might select one of the two semantically related items. This strategy would yield a target response on half of the context-consistent trials, but would always produce an error in context-inconsistent trials.

A posttest guessing correction was used to correct for this possibility. Choice $e$ for the context-inconsistent trials (semantically related to choice $b$ ) would only be selected in preference to choice $d$ or choice $f$ if the participant was favoring the semantic pairs. Therefore, the difference between the number of choice $e$ errors and a mean of choice $d$ and choice $f$ errors approximates the effect of this guessing strategy on all semantic pair choices $(G)$ :

$\mathrm{G}=$ Response $_{e}[\mathrm{Out}]-$ Response $_{d}[\mathrm{Out}]+$ Response $\left._{f}[\mathrm{Out}]\right) / 2$

The appropriate correction was made by subtracting $\mathrm{G}$ from the number of choice $a$ (hit) and choice $c$ (error) responses in the context-consistent condition, and from the number of choice $b$ (error) and choice $e$ (error) responses in the context-inconsistent condition (see Figure 3).

A similar correction was used to calculate and remove the effects of response bias, which would occur if participants based their choices on recognizing the context independently of the target object. Choice $c$ for the context-inconsistent trials (semantic error related to the context) would only be selected over choice $d$ or choice $f$ because of a bias based on semantic information from the context. The difference between the number of choice $c$ errors and the mean of choice $d$ and choice $f$ errors provided an approximation for the response bias (B):

$$
\left.\mathrm{B}=\text { Response }_{c}[\mathrm{Out}]-\text { Response }_{d}[\mathrm{Out}]+\text { Response }_{f}[\text { Out }]\right) / 2
$$

The appropriate corrections were then made by subtracting B from choice $c$ on the context-inconsistent trials, and $\mathrm{B} / 2$ from choices $a$ and $c$ on the context-consistent trials (because the response bias would be divided between the two alternatives - see Figure 4).

\section{Design}

Four images from each of 33 of the context groups were used to provide 132 target objects. (Context Group 34 was created only to provide nontargets for Context Group 33.) Each object served as a target only once, although each target also appeared as a nontarget in 3 other trials. Within participants, context type was manipulated so that half of these targets were viewed with a consistent context and half with an inconsistent context. The trials within these halves were divided evenly across three context-target SOAs. Six groups of participants were presented stimuli from different sets (1-6). To ensure that targets were presented at each SOA and context type an 


Response
\begin{tabular}{|l} 
a) Target \\
b) Perceptual Error \\
C c) Semantic Error \\
d) Error Type 3 \\
e) Error Type 4 \\
f) Error Type 5
\end{tabular}

\section{Response}

c) Semantic Error

- e) Error Type 4

f) Error Type 5

\author{
Context Consistent \\ Cards - G \\ Paper Fan \\ Chess Board - G \\ Net Curtains \\ Drum \\ Wheel
}

\author{
Context Inconsistent \\ Cards \\ - Paper Fan - G
Pear \\ Conical Flask \\ - Chopsticks - G \\ Drumsticks
}

\begin{abstract}
Figure 3. The guessing correction formula, and how it would be applied to one half of a context-consistent and context-inconsistent pairing. In this example, the target object to be identified is a set of playing cards. In the context-consistent condition, it appears among other objects associated with games, and in the context-inconsistent condition, it appears among different types of fruit.
\end{abstract}

equal number of times, these were divided into two blocks of 66 trials. Block order was balanced across participants. The study was a mixed design, with 2 within-participants variables $(2 \times 3)$ and 2 between-participants variables $(6 \times 2)$. Stimuli were created in pairs, each including a context-consistent and a context-inconsistent version (see Figures 3 and 4). For each trial, the perceptually similar $6 \mathrm{AFC}$ choice was matched to the target, and the remaining $6 \mathrm{AFC}$ choices were matched to the context, so that the choices offered were identical in the consistent and inconsistent context lists. Participants completed 16 practice trials on nonexperimental stimuli.

\section{RESULTS}

Table 1 displays the mean error rates across context conditions before and after the response bias correction. (The guessing correction has been made on both sets of results.) Error rates are lower in the context-consistent condition than in the context-inconsistent condition regardless of the response bias correction, but the correction reduces the magnitude of this difference. This reduction most strongly affected the number of trials in which the nontarget object name semantically related to the target was chosen (see Figure 5). Error rates were subject to a repeated measures ANOVA with factors of context type (consistent vs. inconsistent) and SOA ( $-104 \mathrm{msec}$, $-52 \mathrm{msec}, 0 \mathrm{msec}$ ), and between-subjects factors of stimulus set and block order. The contextual facilitation prior to the response bias correction was significant $[F(1,48)=$ $14.61, p<.01]$. Furthermore, despite a reduction in the effect, the contextual facilitation was also significant postcorrection $[F(1,48)=8.41, p<.01]$.

A main effect was also found for $\operatorname{SOA}[F(2,96)=50.92$, $p<.01$ ], with the $-104-\mathrm{msec}$ display condition performing worst $(43.4 \%)$, followed by the -52 -msec display condition $(33.4 \%)$, and then the simultaneous condition
(28.7\%). In addition, there was a significant interaction between context type and SOA $[F(2,96)=3.37, p<.01]$ before response bias correction that highlighted an increasing contextual facilitation for the context-consistent condition with the earlier context displays. Post hoc analysis of each SOA using a mixed design ANOVA revealed significant effects of context type for $-104 \mathrm{msec}[F(1,48)=$ $13.64, p<.01]$ and $-52 \operatorname{msec}[F(1,48)=8.71, p<.01]$ but not for $0 \mathrm{msec}[F(1,48)=1.37, p>.1]$. No significant interaction was found between context type and SOA after bias correction.

Error type was introduced as a third variable in a repeated measures ANOVA (with context type and SOA) controlled by between-subjects variables of order and session set. This analysis included only data from trials with incorrect responses from the 6AFC task (see Table 2 and Figure 5). A main effect of error type was found $[F(4,192)=34.30, p<.01]$, and post hoc analysis using the Bonferroni method revealed significantly more perceptual errors than any other error type, and more semantic errors than control errors (Types 3, 4, and 5-see Figures 3 and 4, above). Types 3, 4, and 5 did not differ significantly between themselves.

There was a significant interaction between error type and SOA $[F(8,384)=5.45, p<.01]$ that highlighted the graded increase of semantic errors when nontargets were displayed prior to the target. There was a trend suggesting an interaction between error type, SOA, and context that implied that semantic errors were more strongly affected by SOA during the context-inconsistent condition $[F(8,384)=1.97, p=.11$ Greenhouse-Geisser $]$; however, this interaction was not significant.

Are the context effects due to semantic consistency, or could they be due to higher perceptual similarity within

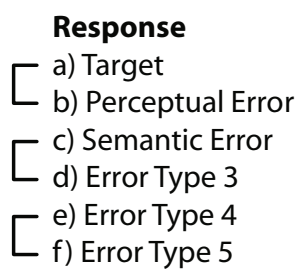

Figure 4. The response bias correction formula, and how it would be applied to the other half of the context pairing presented in Figure 3. This figure also illustrates how the 6AFC design counterbalanced list items between context group pairs. 
Table 1

Mean Error Rates (\%) of Participants in Each Condition

\begin{tabular}{|c|c|c|c|c|c|c|}
\hline \multirow{3}{*}{$\begin{array}{l}\text { Bias Correction } \\
\text { and Context Type }\end{array}$} & \multicolumn{6}{|c|}{ Context SOA } \\
\hline & \multicolumn{2}{|c|}{$-104 \mathrm{msec}$} & \multicolumn{2}{|c|}{$-52 \mathrm{msec}$} & \multicolumn{2}{|c|}{$0 \mathrm{msec}$} \\
\hline & $M$ & $S E$ & $M$ & $S E$ & $M$ & $S E$ \\
\hline \multicolumn{7}{|l|}{ Uncorrected for Bias } \\
\hline In context & 36.10 & 2.4 & 30.00 & 2.5 & 27.24 & 2.7 \\
\hline Out of context & 45.01 & 3.1 & 35.46 & 2.8 & 29.47 & 2.9 \\
\hline \multicolumn{7}{|l|}{ Corrected for Bias } \\
\hline In context & 32.30 & 2.5 & 28.77 & 2.6 & 26.99 & 2.8 \\
\hline Out of context & 37.39 & 3.0 & 33.07 & 2.8 & 28.98 & 3.1 \\
\hline
\end{tabular}

the context groups? Eight participants rated the perceptual similarity between target and nontarget objects on a scale of 1-6. Targets were rated more perceptually similar to the nontargets in the consistent condition (2.46) than in the inconsistent condition $(1.91)(t=11.72, p<.01)$. For each target, the effect of context on accuracy was calculated by subtracting accuracy in the context-inconsistent condition from accuracy in the context-consistent condition. Likewise, a perceptual similarity score for each target was calculated by subtracting the rating in the context-inconsistent condition from the rating in the context-consistent condition. A significant negative correlation between them $(r=-.19)$ indicated that perceptual similarity actually hampers contextual facilitation. Thus, the context effect is due to semantic relationships rather than to perceptual similarity.

\section{DISCUSSION}

Our study tested whether contextual facilitation could be explained through response bias alone. Participants made fewer identification errors when the target was semantically related to the contextual nontargets than when it was unrelated, indicating a facilitatory effect of context. This context effect remained significant even after correction for response bias. In sum, there is an influence of nontargets on perceptual processes during target recognition that is not accounted for by functional isolation.

Three key issues are highlighted by this result: (1) the nature of the context effect, (2) the generalizability between object arrays and naturalistic scenes, and (3) the implications for object-recognition models. These findings imply an integration of perceptual information from the target and semantic knowledge from the context prior to the target identification. However, this integration, which is synonymous with a superiority effect, does not explain the entire context effect. Response bias in context effects has been noted before (see, e.g., Biederman, 1981). In this study, as in previous studies, a portion of the context effect was due to response bias, and this bias needs to be explored to fully understand the influence of context on recognition.

The response biases found here are related to the SOA between nontargets and targets. The increase in error rates in nonsimultaneous SOAs may be a result of participants being unable to immediately disengage from attending to the nontargets when the target appears (Chun \& Potter, 1995), which would reduce perceptual information gained from the target and thus delay target identification. In addition, dur- ing the -104-msec condition, and to a lesser degree in the -52-msec condition, participants had more time to process contexts held in iconic memory. The lack of a context effect at the simultaneous presentation suggests that more time was needed to extract and utilize semantic information from context in both response bias and superiority effects. However, context effects have been found at $65 \mathrm{msec}$ (Auckland, 2005) and $80 \mathrm{msec}$ (Davenport \& Potter, 2004), suggesting that the display layout used here may have slowed encoding of the context somewhat. Our findings indicate that the superiority effect develops faster but remains constant, whereas the response bias effect increases with time, target interference, or both. Precisely how these processes interact merits further investigation. The fact that the context was visible for a time without the target, combined with the attentional cue
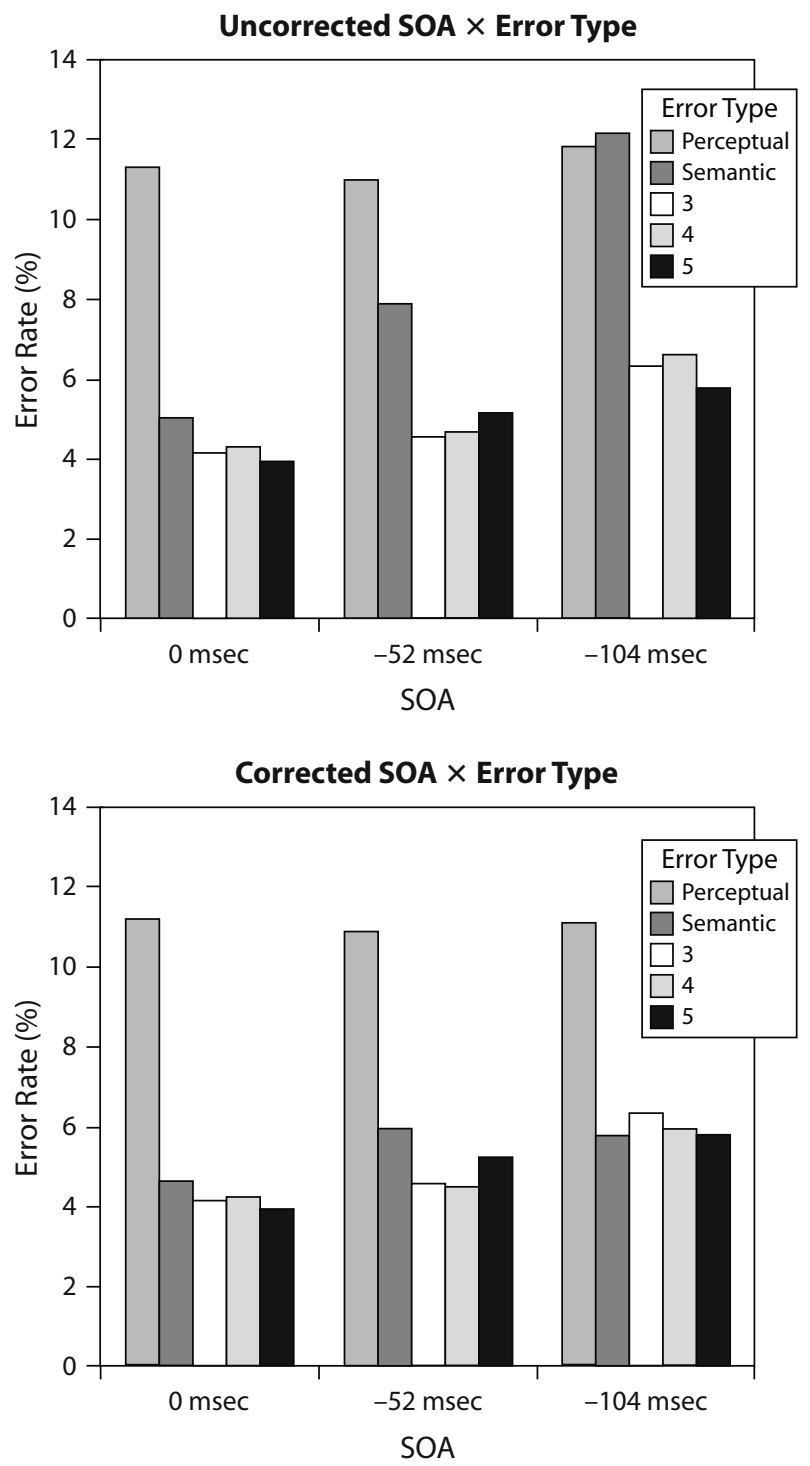

Figure 5. Graphs showing error rates broken down by error type before bias correction (left) and after bias correction (right). Bar 1 represents perceptual errors and Bar 2 represents semantic errors. 
Table 2

Mean Error Rates (\%) of Participants, Broken Down by Error Type

\begin{tabular}{lrrrrr}
\hline & \multicolumn{4}{c}{ Context } \\
\cline { 2 - 3 } Context $\times$ Error Type & \multicolumn{2}{c}{ In Context } & & \multicolumn{2}{c}{ Out of Context } \\
\cline { 2 - 3 } \cline { 5 - 6 } & $M$ & $S E$ & & $S$ & $S E$ \\
\hline Perceptual errors & 10.10 & 0.73 & & 11.98 & 0.80 \\
Semantic errors & 5.54 & 0.98 & & 5.29 & 0.47 \\
Error Type 3 & 4.80 & 0.42 & & 5.20 & 0.55 \\
Error Type 4 & 4.42 & 0.42 & & 5.29 & 0.47 \\
Error Type 5 & 4.50 & 0.57 & & 5.38 & 0.50 \\
\hline
\end{tabular}

to bring attention to the context locations, may provide part of the explanation for why context effects appeared in this experiment but not in some previous tests.

This study demonstrates that semantic relationships between objects in an array are sufficient to generate contextual effects. (See also Henderson, Pollatsek, \& Rayner, 1987.) It seems likely that our results will generalize from arrays of isolated objects to naturalistic scenes (but see Boyce \& Pollatsek, 1992). Contextual facilitation gained through appreciating the semantic consistency of objects would offer advantages in the "real" world, and hence be more likely to have evolved through looking at naturalistic scenes. As such, it would be strange for the interaction between perceptual and semantic knowledge to exist with object arrays and be lost for naturalistic scenes, rather than to have developed for scenes and transferred to object arrays. We expect that the effects demonstrated here will arise whenever multiple objects are visible and attended together. Although additional effects could be present when the objects form a coherent scene, we now have a basis by which to examine these and identify how scene-configuration factors influence contextual facilitation. Biederman (1981) has proposed that the objects within a scene can be processed like an object array. To have different mechanisms (functional isolation and process integration) for specific classes of context (naturalistic scenes and object arrays) would seem more complex than to have a base mechanism shared by both, on which a second mechanism may or may not exist to handle configuration information.

The functional isolation hypothesis has provided objectrecognition models with a theoretical premise for not accounting for visual context effects in recognition tasks. If nontarget stimuli did not influence perceptual processes or representation, they could be placed outside the recognition framework. Consequently, most object-recognition models deal poorly, or not at all, with multiple-object stimuli. This study has demonstrated that the functional isolation hypothesis cannot fully explain contextual effects. The results are consistent with a criterion modulation hypothesis, but also with other views (e.g., Bar, 2004; Boyce \& Pollatsek, 1992). Models of object recognition will need to consider these alternative views and the impact of nontarget influences on perceptual processes.

\section{AUTHOR NOTE}

This work was supported by an award from the U.K. Economic and Social Research Council to M.E.A. We thank Martin Batty, Irving Biederman, and Andrew Hollingworth for suggestions, and Fabiola Gomes-Pinto for assistance in conducting the experiment. Correspondence concerning this article should be addressed to K. R. Cave, Department of Psychology, University of Massachusetts, Tobin Hall, Amherst, MA 01003 (e-mail: kcave@, psych.umass.edu).

Note-Accepted by David A. Balota's editorial team.

\section{REFERENCES}

AucKLAND, M. E. (2005). The influence of visual context on object recognition. Unpublished doctoral dissertation, University of Southampton.

BAR, M. (2004). Visual objects in context. Nature Reviews Neuroscience, 5, 617-629.

Biederman, I. (1981). On the semantics of a glance at a scene. In M. Kubovy \& J. R. Pomerantz (Eds.), Perceptual organization (pp. 213263). Hillsdale, NJ: Erlbaum.

Boyce, S. J., \& PollatSeK, A. (1992). Identification of objects in scenes: The role of scene background in object naming. Journal of Experimental Psychology: Learning, Memory, \& Cognition, 18, 531-543.

Chun, M. M., \& PotTer, M. C. (1995). A two-stage model for multiple target detection in rapid serial visual presentation. Journal of Experimental Psychology: Human Perception \& Performance, 21, 109-127.

Davenport, J. L., \& PotTer, M. C. (2004). Scene consistency in object and background perception. Psychological Science, 15, 559-564.

Friedman, A. (1979). Framing pictures: The role of knowledge in automatized encoding and memory for gist. Journal of Experimental Psychology: General, 108, 316-355.

Henderson, J. M., Pollatsek, A., \& Rayner, K. (1987). Effects of foveal priming and extrafoveal preview on object identification. Journal of Experimental Psychology: Human Perception \& Performance, 13, 449-463.

Hollingworth, A., \& Henderson, J. M. (1998). Does consistent scene context facilitate object perception? Journal of Experimental Psychology: General, 127, 398-415.

HolLingworth, A., \& Henderson, J. M. (1999). Object identification is isolated from scene semantic constraint: Evidence from object type and token discrimination. Acta Psychologica, 102, 319-343.

JONIDES, J. (1981). Voluntary versus automatic control over the mind's eye's movement. In J. [B.] Long and A. [D.] Baddeley (Eds.), Attention and performance $I X$ (pp. 187-203). Hillsdale, NJ: Erlbaum.

KossLyn, S. M. (1994). Image and brain: The resolution of the imagery debate. Cambridge, MA: MIT Press.

MüLLER, H. J., \& RABbitT, P. M. A. (1989). Reflexive and voluntary orienting of visual attention: Time course of activation and resistance to interruption. Journal of Experimental Psychology: Human Perception \& Performance, 15, 315-330.

PaLmer, S. E. (1975). The effects of contextual scenes on the identification of objects. Memory \& Cognition, 3, 519-526.

Turatto, M., Benso, F., Facoetti, A., Galfano, G., Mascetti, G. G., \& Umiltà, C. (2000). Automatic and voluntary focusing of attention. Perception \& Psychophysics, 62, 935-952.

\section{ARCHIVED MATERIALS}

The following materials associated with this article may be accessed through the Psychonomic Society's Norms, Stimuli, and Data archive, www.psychonomic.org/archive.

To access these files, search the archive for this article using the journal name (Psychonomic Bulletin \& Review), the first author's name (Auckland), and the publication year (2007).

FILE: AUCKLAND_PBR_2007.zip

DESCRIPTION: The compressed archive file contains one file, AucklandCave\&Donnelly_Stimuli, containing a table of the stimuli used in the testing of context on object recognition.

AUTHOR’s E-MAIL ADDRESS: kcave@psych.umass.edu. revision accepted for publication June 8,2006 .) 